\title{
A LEGISLAÇÃO BRASILEIRA SOBRE DIREITO AMBIENTAL ATÉ 2010 E SUAS IMPLICAÇÕES PARA AS TECNOLOGIAS DA INFORMAÇÃO E COMUNICAÇÃO (TIC) SUSTENTÁVEIS ${ }^{1}$
}

\section{BRAZILIAN LEGISLATION ENVIRONMENTAL LAW UNTIL 2010 AND ITS IMPLICATIONS FOR THE INFORMATION TECHNOLOGY AND COMMUNICATION (ICT) SUSTAINABLE}

\author{
Data do recebimento do artigo: 27/04/2014 \\ Data do aceite do artigo: 9/9/2014 \\ Data da publicação: 8/12/2014 \\ Processo de Avaliação: Double Blind Review
}

Samuel de Barros Moraes ${ }^{2}$

Graduado em Engenharia Elétrica - Faculdade de Engenharia São Paulo Mestrando Profissional em Gestão e Tecnologia em Sistemas Produtivos

Centro Paula Souza

\section{Celi Langhi}

Doutora em Psicologia - Universidade de São Paulo

Professor do Mestrado Profissional em Gestão e Tecnologia em Sistemas Produtivos

Professora do Centro Paula Souza

\section{Marcos Crivelaro}

Pós-Doutor - Instituto de Pesquisas Energéticas e Nucleares

Doutor em Engenharia de Materiais -- Instituto de Pesquisas Energéticas e Nucleares.

Professor do Centro Paula Souza

\section{RESUMO:}

Este artigo faz uma análise da legislação ambiental brasileira, visando identificar suas referências à tecnologia da informação e dentro de suas exigências legais como ela influência a adoção de formas sustentáveis de uso da tecnologia da informação pelas empresas. Para tanto se procede a uma revisão bibliográfica da legislação promulgada até 2010, localizando-se as menções diretas e indiretas a tecnologia e analisando o contexto em que cada menção foi feita. A análise realizada indica que TIC é considerada pela legislação uma ferramenta fundamental para a preservação ambiental e como causadora de baixo impacto ambiental, dado que existem poucas referências as TIC neste sentido.

\section{Palavras-chave:}

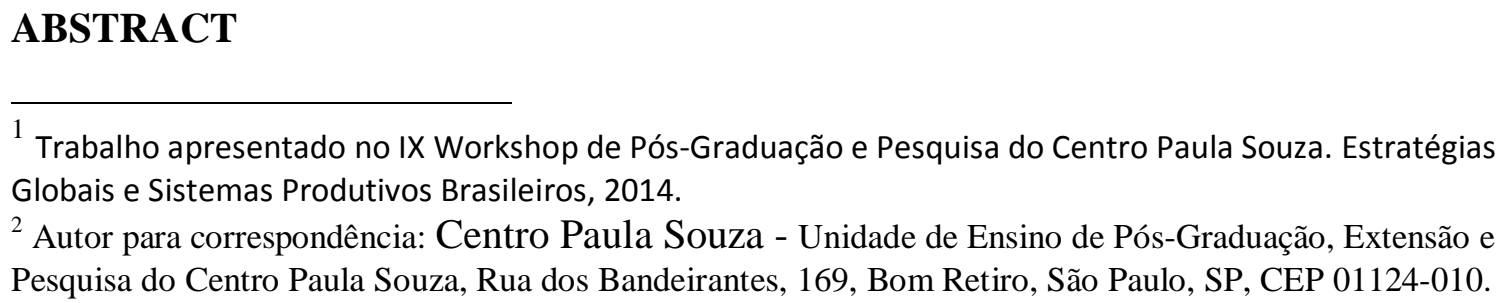


This article does an analysis of the Environmental Brazilian legislation, in order to identify the references to information technology and, between their legal requirements, how the legislation influences the adoption of sustainable ways of information technology usage by companies. For this purpose, a bibliography revision was made of the legislation promulgated until 2010, localizing the direct and indirect mentions of technology and analyzing the context in which mention was made. The analysis realized indicate that ICT is considered by the legislation as a fundamental tool for the Environmental preservation and as cause of low environmental impact, since there are few references to it accordingly.

Keywords: Green IT. Green IS. Environmental Law. Information Technology. 


\section{INTRODUÇÃO}

Considerando que o impacto da ação humana sobre o meio ambiente normalmente resulta em sua deterioração, nos últimos anos tem-se visto uma crescente preocupação com a utilização dos recursos naturais. Isso tem se tornado cada vez mais presente no dia-a-dia das empresas, pessoas e governos, com a utilização de normas técnicas, ações empresariais e leis, que afetam a forma como se deve agir em nosso cotidiano, seja nos aspectos de consumo, deslocamento, alimentação ou uso de tecnologia (PONIATWOSKI, 2010).

Por outro lado, a Tecnologia da Informação e Comunicação (TIC) está presente na grande maioria das empresas, tendo seu uso disseminado em todos os níveis hierárquicos, como apoio aos negócios, e auxiliando os objetivos organizacionais (NREL, 2011). No Brasil, a pesquisa sobre o uso das TIC do CGI.BR ${ }^{3}$ de 2012 constatou que o seu uso está presente em "praticamente todas as empresas brasileiras com 10 ou mais pessoas utilizando-a: 98\% das empresas possuem computador e 97\% delas acessam a Internet" (NIC.BR, 2013, p. 211).

Com a identificação da penetração das TIC nos negócios e na vida pessoal de forma crescente, a comunidade profissional e científica direcionou sua atenção para os efeitos do seu uso no meio ambiente (SARTORI, ENSSLIN, et al., 2014).

Este contexto, também é influenciado pela legislação ambiental, que diferente de outras legislações, não regula apenas a relação entre as pessoas, mas também a relação entre as pessoas e o meio ambiente e ainda promovendo ajustes nas relações sociais que fazem a intermediação com o meio ambiente. Os alvos destas leis são os governos, as organizações não governamentais, as empresas e os cidadãos, em relação a seus direitos e obrigações com o meio ambiente, incluindo florestas, uso da água, uso do solo, poluição, vida selvagem, recursos minerais e ainda o planejamento de uso e proteção dos recursos naturais. Em resumo pode-se entender que a legislação ambiental trata de comportamento (YONGMEI, 2013).

Assim torna-se relevante o entendimento de como a legislação ambiental influencia o uso, o desenvolvimento e a aplicação das TIC, impondo adaptações às empresas. Para tanto se procede a uma revisão bibliográfica da legislação brasileira sobre meio ambiente, publicada pelo Congresso Nacional Brasileiro contemplando as leis promulgadas de 1965 a 2010 (CONGRESSO BRASILEIRO, 2010), visando identificar as referências sobre as TIC e a adoção da TI Verde pelas empresas.

\footnotetext{
${ }^{3}$ Comitê Gestor da Internet no Brasil
} 


\section{TECNOLOGIA DA INFORMAÇÃO}

A sustentabilidade no uso das TIC tem emergido na área científica e profissional envolvida com seu uso e desenvolvimento. Existem muitos estudos que sugerem práticas de gestão ambiental para a utilização consciente das TIC. Esse impulso pode ter sido provocado pelo crescimento econômico, pelas potenciais crises econômicas, pela preocupação com a disparidade de riqueza e pela expectativa de esgotamento dos recursos naturais, representando uma urgência na compatibilização entre o capital ambiental, o capital econômico e o capital social (SARTORI, ENSSLIN, et al., 2014).

Como motivador para a adoção de práticas sustentáveis em TIC, pode-se identificar duas linhas de benefícios ou de ganhos relacionados às práticas, na grande maioria dos textos analisados pelos autores, a primeira delas relacionada ao efeito direto proporcionando redução de consumo de energia e redução de despesas financeiras a segunda a efeitos indiretos, com uma perspectiva estratégica e relacionados a aspectos subjetivos, como melhoria de imagem, aderência à legislação ou posicionamento frente à concorrência com a geração de diferenciais (HIRD, 2008)(KANE, 2010)(PONIATWOSKI, 2010).

Mesmo com a expectativa da aplicação de práticas de TIC sustentáveis se concentrando na redução dos custos associados ao consumo de energia e redução do impacto ambiental da empresa, identificam-se em alguns autores outros pontos importantes, que não recebem a devida atenção, que se tornam benefícios estratégicos para a organização em termos de criação de valor para os clientes, para o negócio e para a sociedade (HARMON e AUSEKLIS, 2009) com o uso das TIC sustentável como ferramenta de inovação (NIDUMOLU, PRAHALAD e RANGASWAMI, 2011).

A utilização do conceito de TIC sustentável se tornou comum após a publicação, pelo instituto de pesquisa empresarial Gartner Group, do estudo chamado Green IT: a new industry shock wave (MINGAY, 2007), onde é definido o conceito de Green IT como "o uso ótimo da Tecnologia da Informação para o gerenciamento da sustentabilidade ambiental na organização e na sua cadeia de suprimentos, bem como de seus produtos, serviços e recursos, durante todo seu ciclo de vida” (MINGAY, 2007, p. 1).

Desde então diversos projetos foram conduzidos e estudos seminais publicados por associações industriais como a Global e Sustainability Initiative ${ }^{5}$ (GeSI) em 2008, por organizações não governamentais como a World Wide Fund for Nature ${ }^{6}$ (WWF) também em 2008 e organizações internacionais como a Organização para a Cooperação e Desenvolvimento Económico (OCDE) em 2010. A maioria destes estudos se refere ao impacto da TI como emissorana emissão de gases de efeito estufa, ou relacionados à energia elétrica consumida ou ainda como tecnologia capaz de gerenciar processos de conservação de energia em diversos segmentos de negócios (HILTY, LOHMANN e HUANG, 2011).

Alguns autores tratam este tema com uma nomenclatura mais específica, considerando Green IS (Green Information Systems) ${ }^{7}$ para o que se refere ao uso de TI como ferramenta para a sustentabilidade, ou seja, foco na aplicação da tecnologia para

\footnotetext{
4 TI Verde: uma nova revolução na indústria

${ }^{5}$ Iniciativa Global para Sustentabilidade Digital

${ }^{6}$ Fundo Mundial para vida selvagem

${ }^{7}$ Sistemas de Informação Verde
} 
resolver problemas ambientais, enquanto o termo Green IT (Green Information Technology) ${ }^{8}$ é reservado para o uso de TI que busca reduzir o impacto no meio ambiente (OZTURK, UMIT, et al., 2011)e (WATSON, BOUDREAU e CHEN, 2010). A figura1 apresenta de forma visual a nomenclatura proposta.

Figura 1- Diferenciação do uso de tecnologia sustentável

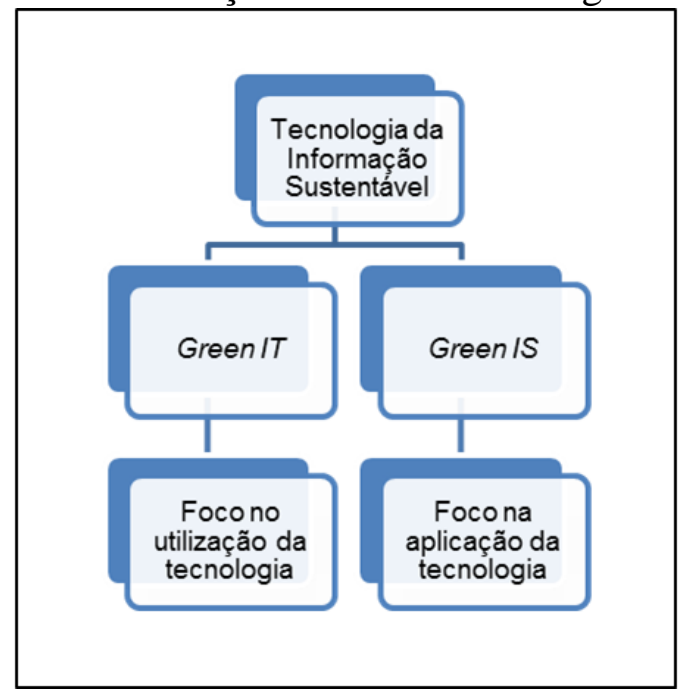

Fonte: Elaborado pelos autores a partir de (OZTURK, UMIT, et al., 2011)

Mesmo não se identificando pressões políticas para o uso da TIC para a sustentabilidade, existem pesquisas que terão um papel crucial no desenvolvimento e aplicação de conceitos de sustentabilidade na sociedade (HILTY, LOHMANN e HUANG, 2011).

\section{LEGISLAÇÃO AMBIENTAL}

No cenário tecnológico atual, ainda concorrem às ações governamentais, na forma de leis, como impulsionadoras adicionais ao processo em curso de revisão da relação entre empresa e meio ambiente. O desenvolvimento da legislação relacionada ao meio ambiente se dá a partir de 1992, quando diversos países passaram a tratar este tema como um problema comum. Sob a coordenação das Nações Unidas foi apresentado o documento intitulado United Nations Framework Convention on Climate Change e para sua análise foi convocada a II Conferência das Nações Unidas sobre Meio Ambiente e Desenvolvimento Humano, realizada em 1992 no Rio de Janeiro, o debate teve como principal tema a discussão sobre o desenvolvimento sustentável e como reverter o processo de degradação ambiental. (NAÇÕES UNIDAS, 1992).

O resultado desta conferência deu origem a um acordo global em reposta às mudanças climáticas, com regras e mecanismos de ação mais elaborados e detalhados. Definindo objetivos de redução de emissão de gases de efeito estufa pelos países desenvolvidos e os

\footnotetext{
${ }^{8}$ Tecnologia da Informação Verde

${ }^{9}$ Painel das Nações Unidas para a Convenção da Mudança Climática
} 
mecanismos de compensação. Este acordo, chamado de Protocolo de Quioto, foi celebrado em 1997, na cidade de mesmo nome no Japão (NAÇÕES UNIDAS, 1998). Este protocolo continua ratificado por 192 países, apesar de apenas 36 países o seguirem após a conferência de Doha de 2012; mas existe uma previsão de ampliação da quantidade de países comprometidos até a conferência programada para 2015 (UNITED NATIONS FRAMEWORK CONVENTION ON CLIMATE CHANGE, 2014).

Em relação ao Brasil, ele se posiciona como um país avançado nos aspectos relacionados à legislação ambiental, muito em consequência dos tratados e convenções internacionais de que é signatário. Sendo reconhecido por seu engajamento na proteção ambiental, que foi demonstrado nos principais fóruns internacionais que ele participou (GUEDES, 2014).

Vê-se esta proeminência na promulgação, em 1981, da Política Nacional do Meio Ambiente, através da Lei 6.938/81, onde foram estabelecidos os objetivos nacionais com relação à preservação da natureza, esta política considera o imperativo de se conciliar o desenvolvimento econômico com a preservação ambiental e a qualidade de vida (CONGRESSO BRASILEIRO, 2010). Indicando que o meio ambiente deve ser protegido, definindo as regras gerais, os princípios e a conduta que deve ser adotada e implantada por todos os níveis governamentais, reforçando a responsabilidade compartilhada dos governos federal, estadual e municipal de acordo com sua capacidade financeira e sua abrangência administrativa (GUEDES, 2014). Esta mesma Lei criou o Sistema Nacional de Meio Ambiente, integrado por um órgão colegiado: o Conselho Nacional do Meio Ambiente (CONAMA), órgão consultivo e deliberativo do Sistema Nacional do Meio Ambiente SISNAMA, demonstrando o avanço do Brasil, nos aspecto legais, com relação a preservação do meio ambiente.

A preocupação ambiental se manifesta também na Constituição da República Federativa do Brasil de 1988, conforme artigo do capítulo V relacionado ao meio ambiente:

“Art. 225: Todos têm direito ao meio ambiente ecologicamente equilibrado bem de uso comum do povo e essencial à sadia qualidade de vida, impondo-se ao Poder Público e à coletividade o dever de defendê-lo e preservá-lo para as presentes e futuras gerações.

V - controlar a produção, a comercialização e o emprego de técnicas, métodos e substâncias que comportem risco para a vida, a qualidade de vida e o meio ambiente.” (CONGRESSO NACIONAL BRASILEIRO, 1988, p. 36)

Esta diretriz constitucional demonstra a preocupação do Brasil com relação ao meio ambiente e impondo-se como uma necessidade de ação coletiva na sua preservação (BRITO e BRITO, 2012). Esta preocupação com a natureza na Constituição brasileira é anterior inclusive às grandes manifestações internacionais relacionadas à proteção do meio ambiente.

Ainda com relação ao arcabouço legal brasileiro, tem-se o Plano Nacional sobre Mudança do Clima no Brasil promulgado através do Decreto $n^{\circ}$ 6.263, de 21 de novembro de 2007, que tem por objetivo identificar, planejar e coordenar ações e medidas que permitam mitigar as emissões de gases de efeito estufa geradas no Brasil e ainda promover e definir as ações necessárias à adaptação da sociedade aos possíveis impactos devido à mudança do clima. 
O conjunto brasileiro de leis relacionadas ao meio ambiente, com base nas definições técnicas apresentadas, passa a ser analisado nos próximos capítulos deste artigo.

\section{MÉTODO}

Procedeu-se uma leitura crítica na legislação brasileira nos iténs relacionados ao meio ambiente buscando-se referências ligadas aos TIC. Considerando a tecnologia como algo possível de ser usado de maneira sustentável no cotidiano das pessoas e das empresas.

Foram realizadas buscas (pesquisas) a procura de palavras correlacionadas à Tecnologia da Informação.

A pesquisa consistiu na identificação de palavras associadas a tecnologia da informação, considerando seus sinônimos mais comuns, e ainda foi tratado igualmente menções no singular e no plural. Pesquisou-se também as palavras tecnologia, informação, dado, como forma alternativa a informação, telecomunicação e rede ou comunicação, para se denotar as telecomunicações, além das expressões tecnologia da informação e rede de comunicação. Encontrando-se a distribuição apresentada na tabela 1, que sumariza o resultado das buscas nos documentos legais.

Tabela 1 - Palavras relacionadas a Tecnologia da Informação e suas menções na Legislação

\begin{tabular}{lcc}
\hline \multicolumn{1}{c}{ Termo } & $\begin{array}{c}\text { Número de menções } \\
\text { total }\end{array}$ & Relacionadas as TIC \\
Tecnologia da informação & 0 & 0 \\
Tecnologia & 40 & 16 \\
Informação/Informações & 124 & 40 \\
Dado/Dados & 47 & 8 \\
Telecomunicação/Telecomunicações & 2 & 1 \\
Comunicação/Comunicações & 12 & 0 \\
Rede de Comunicação/Rede & 16 & 2 \\
Rede de & 0 & 0 \\
Comunicações & & \\
\hline
\end{tabular}

Fonte: Pesquisa dos autores

O contexto onde as palavras estavam presentes foi analisado para se identificar a que se referia cada utilização. Fazendo se uma separação analítica para citações diretamente relacionadas aos temas Green IT e Green IS, além da identificação de outras citações onde se poderiam entender menções indiretas ao assunto sustentabilidade relacionada á TIC, contabilizando as palavras que estavam sendo usadas em um contexto referente a assuntos distintos e ainda identificando as mesmas palavras quando faziam parte de títulos de leis e de capítulos. 


\section{RESULTADOS E DISCUSSÃO}

Uma análise contextual em todas as menções dos termos usualmente referentes às TIC na legislação ambiental, conforme pode ser observado no gráfico 1, indica em grande parte das vezes que eles aparecem se referem a tecnologias em geral com uso em sustentabilidade, como tratamento de água de redução de poluentes atmosféricos dentre as principais, e não especificamente ao uso sustentável da tecnologia da informação, além do que, muitas vezes eles aparecem nos títulos dos capítulos. Quando as palavras estão associadas à tecnologia da informação, destaca-se o seu maior uso tratando de Green IS, com poucas referências a Green IT e ainda algumas referências genéricas que podem ser aplicadas as TIC sustentáveis. As referências indiretas encontradas na legislação se referem ao descarte de produtos poluentes.

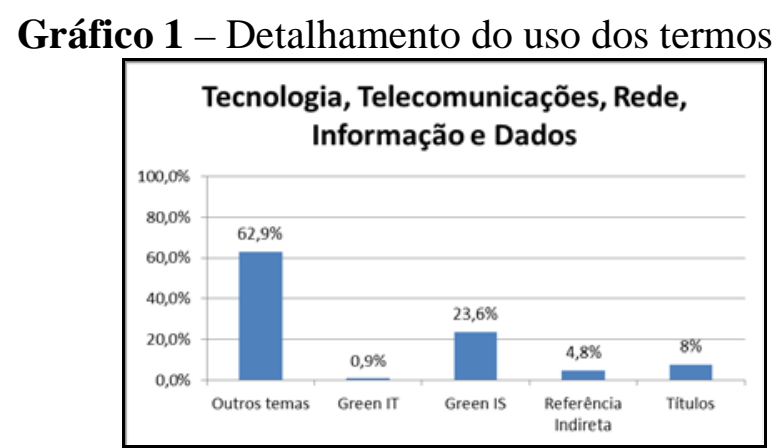

Fonte: análise dos autores

Na revisão contextual da palavra tecnologia, sumarizada no gráfico 2, observa-se que ela é usada para identificar qualquer ferramental disponível para a melhoria do meio ambiente, nesta análise, vê-se a palavra tecnologia de forma abrangente, sendo possível associá-la como referência indireta as TIC. Com menor uso ela é empregada especificamente para tratar de TIC sustentável, seja Green IS ou Green IT.

Gráfico 2 - Detalhamento do uso do termo tecnologia

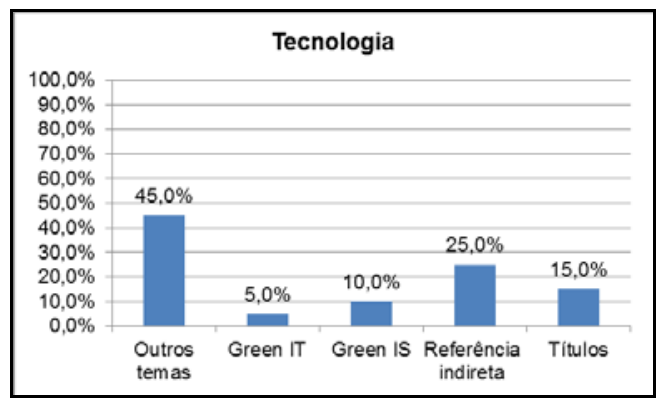

Fonte: análise dos autores

O termo tecnologia, quando aplicado de forma abrangente, onde se pôde incluir TIC, se referenciando à produção e descarte da tecnologia em geral, verificou-se com a Política 
Nacional de Resíduos Sólidos a necessidade de "cooperação técnica e financeira entre os setores público e privado para o desenvolvimento de pesquisas de novos produtos, métodos, processos e tecnologias de gestão, reciclagem, reutilização, tratamento de resíduos e disposição final ambientalmente adequada de rejeitos" (CONGRESSO BRASILEIRO, 2010, p. 494), mesmo não fazendo referência direta a TIC inclui os produtos relacionados. Indicando um esforço no sentido de direcionar a produção para técnicas mais limpas, sem o uso de substâncias com alta toxidade, o que afeta diretamente os fabricantes de equipamentos eletrônicos.

A palavra informação e seu plural, conforme gráfico 3, quando utilizada se apresenta, pelo seu contexto, principalmente relacionada à difusão do conhecimento, quando não está fazendo referencia a Green IS. A palavra dados, conforme gráfico 3, quando sinônimo de informação, tem seu uso majoritariamente relacionado, também, a disseminação da informação, quando associado as TIC, se refere a Green IS, principalmente associada a sistemas de gerenciamento de informações relacionadas ao meio ambiente.

Gráfico 3 - Detalhamento do uso dos termos informação e dados

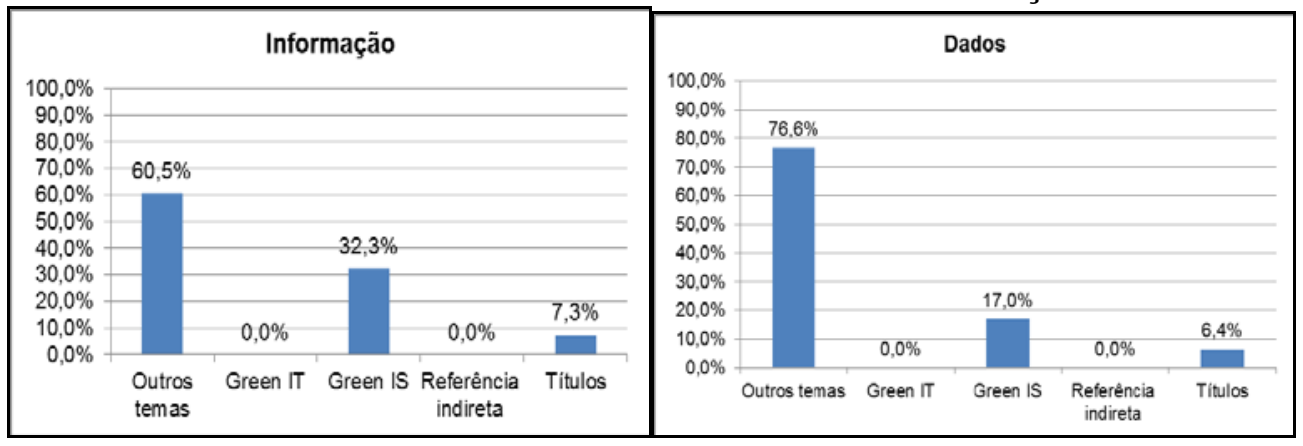

Fonte: análise dos autores

As palavras telecomunicações e rede, como sinônimos de comunicação, normalmente são utilizadas para descrever recursos tecnológicos que permitem a troca de informações à distância entre dois ou mais elementos.

Na legislação, a palavra telecomunicação, conforme gráfico 4, se apresenta dentro do contexto de sua aplicação e se refere aos cuidados que se deve tomar com relação ao impacto ao meio ambiente, esta aplicação do termo foi considerada como uma referência indireta e associada a Green IT. Já a palavra rede, conforme gráfico 4, é usada em duas situações se referindo a tecnologia da informação, uma citando a importância da Internet, rede mundial de computadores, como ferramenta de proteção ambiental e em outra como um conjunto de serviços de coleta e de tratamento de dados sobre o meio ambiente.

Gráfico 4 - Detalhamento do uso do termo rede e telecomunicações
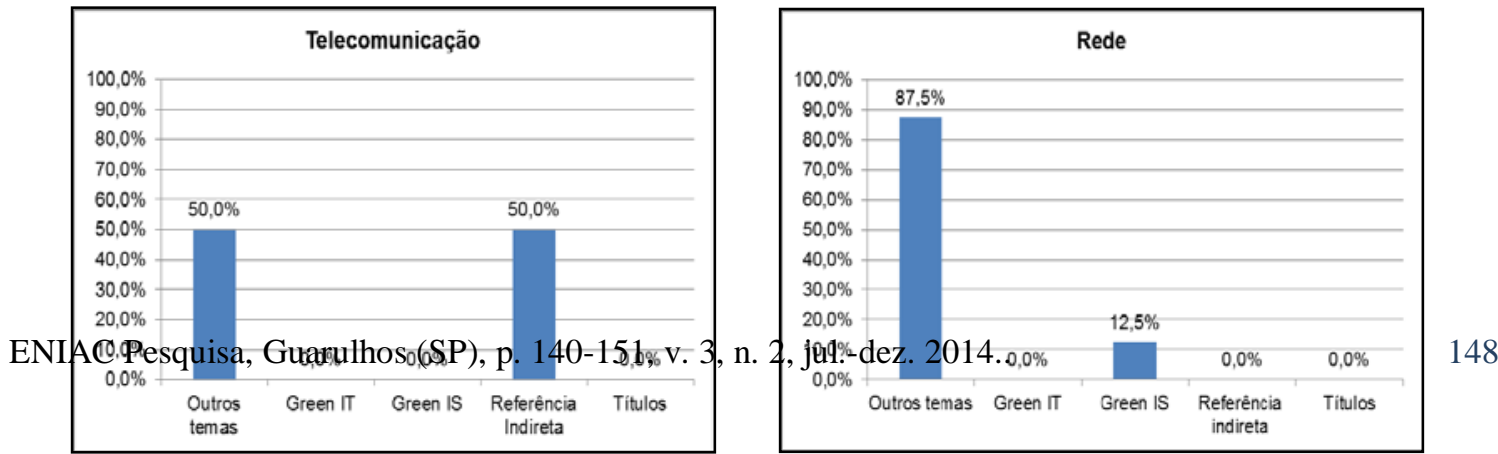
Fonte: análise dos autores

No geral as duas palavras, rede e telecomunicações, normalmente associadas com a vida moderna, pouco aparecem no texto da legislação ambiental, sendo utilizadas em uma porcentagem muito mais baixa que outras palavras relacionadas à tecnologia da informação.

\section{CONSIDERAÇÕES FINAIS}

Com a análise da atual legislação brasileira, foi verificado a não existência especificamente de, uma área de aplicação ou do conhecimento ou mesmo de segmento empresarial, responsável pela preservação do meio ambiente, percebe-se também que o conceito de sustentabilidade atinge todas as áreas do saber e envolve todas as atividades humanas.

Verifica-se que a aplicação da tecnologia da informação, nos diversos documentos legais, se apresenta como uma ferramenta essencial para controlar os processos poluentes, com a proposição de sistemas de gestão de informação que permitam as entidades públicas e privadas o efetivo controle de sua relação com o meio ambiente. Neste contexto se vê claramente o conceito de Green IS, onde a Tecnologia da Informação é proposta como ferramenta para a gestão dos recursos naturais e o controle da ação do homem sobre elas.

O conceito de maior relevância, quando se busca referências na legislação sobre Green IT, se encontra na Política Nacional de Resíduos Sólidos, que define a responsabilidade pelo ciclo de vida dos produtos, indicando que a mesma deve ser compartilhada pelos fabricantes, importadores, distribuidores, comerciantes, consumidores, titulares dos serviços públicos de limpeza urbana e de manejo dos resíduos sólidos, e ainda que as responsabilidades apesar de serem individualizadas, são encadeadas e devem ter o intuito de minimizar o volume de resíduos sólidos e rejeitos gerados, bem como reduzir os impactos causados à saúde e à qualidade ambiental.

Assim como as empresas são impactadas pela necessidade de utilizar a tecnologia da informação para apresentação de dados e relatórios relativos aos impactos ambientais de suas atividades, sua preocupação com o meio ambiente deve crescer. Com relação aos potenciais danos causados pela tecnologia, a legislação impõe às empresas a preocupação com o seu descarte, em conjunto com o descarte de outros produtos poluentes, esta imposição afeta com maior incidência os fabricantes e distribuidores de equipamentos e produtos eletrônicos.

Como a evolução deste artigo será oportuna uma análise equivalente sobre as resoluções do CONAMA e seu impacto sobre o uso da tecnologia da informação pelas empresas. Considerando que este órgão é que define as ações imediatas para a adequação das empresas a processos de preservação do meio ambiente.

\section{REFERÊNCIAS}

BRITO, F. A. A.; BRITO, Á. A. A. A tendência ambientalista da Constituição Federal de 1988. Âmbito Jurídico, Rio Grande, 2012. 
CONGRESSO BRASILEIRO. Legislação Brasileira sobre Meio Ambiente. Brasilia: Edições Câmara, 2010.

CONGRESSO NACIONAL BRASILEIRO. Constituição da República Federativa do Brasil. Brasília: [s.n.], 1988.

GUEDES, M. A. P. Environmental Protection in Brazil: A Matter of Principles. http: //ssrn.com/, 17 abr. 2014. Disponivel em: <http://ssrn.com/abstract=2431490>. Acesso em: 13 maio 2014.

HARMON, R. R.; AUSEKLIS, N. Sustainable IT services: Assessing the impact of green computing practices. Management of Engineering \& Technology, Portland, 2009.

HILTY, L. M.; LOHMANN, W.; HUANG, E. M. Sustainability and ICT - An overview of the field. Notizie di Politeia, 104, 2011.

HIRD, G. Green IT in Pratice. Cambridgeshire: ITGP, 2008.

KANE, G. The Three Secrets of Green Business. Londres: earthscan, 2010.

MINGAY, S. Green IT: The New Industry Shock Wave. Gartner Group. [S.l.], p. 7. 2007.

NAÇÕES UNIDAS. United Nations Framework Convention. Rio de Janeiro. 1992.

NAÇÕES UNIDAS. Kyoto Protocol. Quito, Japão: Nações Unidas, 1998.

NIC.BR. Pesquisa sobre o uso das tecnologias de informação e comunicação no Brasil : TIC Domicílios e Empresas 2012. São Paulo. 2013.

NIDUMOLU, R.; PRAHALAD, C. ; RANGASWAMI, J. P. Why Sustainability Is Now the Key Driver of Innovaion. In: REVIEW, H. B. Greenning your Business Profitably. Boston: Harvard Business Review Publishing Corporation, 2011.

NREL. Department of Energy Laboratories Leadership in Green IT. Golden, Colorado. 2011.

OZTURK, A. et al. Green ICT (Information and Communication Technologies: A Review of Academic and Practitioner Perspectives. Internacional Journal of eBusiness and eGovernment Studies, Istambul, 3, 2011.

PONIATWOSKI, M. Foundation of Green IT. Boston: Prentice Hall, 2010.

SARTORI, S. et al. Mapeamento do estado da arte do tema sustentabilidade ambiental direcionado para a tecnologia de informação. TransInformação, Campinas, 1, 2014.

UNITED NATIONS FRAMEWORK CONVENTION ON CLIMATE CHANGE. Warsaw Outcomes. http: //unfccc.int/, 2014. Disponivel em: <http://unfccc.int/key_steps/warsaw_outcomes/items/8006.php>. Acesso em: 01 ago. 2014. 
WATSON, R. T.; BOUDREAU, M.-C.; CHEN, A. J. Information Systems And Environmentally Sustainable Development: Energy Informatics And New Directions For The IS Community. MIS Quarterly, 34, 2010. 23 - 38.

YONGMEI, X. Study on Value Orientation of Environmental Law. Tianjin, China: Atlantis Press - ICEMSS. 2013. 